\title{
CLINICAL FEATURES AND BIOCHEMICAL PLEURAL FLUID ANALYSIS - VALUABLE TOOLS FOR THE DIAGNOSIS OF TUBERCULOUS PLEURISY
}

\author{
Adelina P. DINUT ${ }^{1,2,3 凶}$, Dragos C. ZAHARIA ${ }^{1,2}$, Stefan DUMITRACHE-RUJINSKI ${ }^{1,2}$, Claudia L. \\ TOMA $^{1,2}$, Miron A. BOGDAN ${ }^{1,2} \bowtie$
}

${ }^{1}$ National Institute of Pneumology "Marius Nasta“, Bucharest, Romania

2 "Carol Davila" University of Medicine and Pharmacy, Bucharest, Romania

${ }^{3}$ Ghencea Medical Center, Bucharest, Romania

Received 03 Nov 2020, Accepted 22 Nov 2020

hitps://doi.org/10.31688/ABMU.2020.55.4.07

\begin{abstract}
Introduction. The infection with Mycobacterium tuberculosis represents a common cause of pleural effusions, and its diagnosis poses difficulties for clinicians. In the attempt to facilitate a quick, accurate and inexpensive diagnosis, the research is focused on evaluating useful biomarkers in the pleural fluid. Adenosine deaminase (ADA) level in the pleural fluid is a biomarker with high specificity and sensitivity for the diagnosis. The objective of the study was to assess the association between ADA levels in the pleural fluid, other pleural fluid characteristics, and clinical aspects in tuberculous pleurisy.
\end{abstract}

Material and methods. We performed a retrospective study on 77 patients diagnosed with tuberculous pleurisy (positive Mycobacterium tuberculosis cultures in the pleural fluid or biopsy specimens and/or specific histological aspect: epithelioid granuloma with caseous necrosis in pleural biopsy), between 2011-2018, in The National Institute of Pneumology "Marius Nasta" Bucharest, Romania.

Results. The ADA values were positively and statistically significant correlated with lactate dehydrogenase

\section{RÉsumé}

Caractéristiques cliniques et analyse des fluides pleuraux biochimiques - des outils précieux dans le diagnostic de la pleurésie tuberculeuse

Introduction. La pleurésie tuberculeuse représente une cause fréquente d'épanchement pleural mais le diagnostic reste un défi pour les cliniciens. Dans le but de faciliter un diagnostic rapide, précis et peu coûteux, la recherche se concentre sur l'évaluation des associations utiles entre les caractéristiques du liquide pleural. Le niveau d'adénosine désaminase (ADA) dans le liquide pleural reste le biomarqueur avec une spécificité et sensibilité élevées.

L'objectif de l'étude était d'évaluer l'association entre les taux d'ADA dans le liquide pleural, d'autres caractéristiques du liquide pleural et les aspects cliniques de la pleurésie tuberculeuse.

Matériel et méthodes. Nous avons réalisé une étude rétrospective sur 77 patients diagnostiqués de pleurésie tuberculeuse (cultures positives de Mycobacterium tuberculosis dans le liquide pleural ou prélèvements de biopsie et / ou aspect histologique spécifique : 
$(\mathrm{LDH})$ and protein levels in the pleural fluid. Also, the mean ADA value was increased in febrile patients as compared with the afebrile ones, statistically significant $(\mathrm{p}<0.05)$.

Conclusions. Probability scores (developed on the ground of biochemical and cytological characteristics of the pleural fluid, clinical and demographic parameters), together with the local epidemiological context, may contribute to a faster, reliable, accessible and non-invasive diagnosis of tuberculous pleurisy.

Keywords: ADA, LDH, tuberculous pleurisy, scoring systems.

\section{Abbreviations list:}

ADA-adenosine deaminase

ESR-erythrocyte sedimentation rate

IFN- $\gamma$ - interferon gamma

$\mathrm{LDH}$ - lactate dehydrogenase

PCR- polymerase chain reaction

PPV- positive predictive value

TB - tuberculosis

SD -standard deviation

\section{INTRODUCTION}

The infection with Mycobacterium tuberculosis is a common cause of pleural effusion in countries with a high prevalence of tuberculosis. Even with new diagnostic tests, that are now available, tuberculous pleurisy remains a real challenge for the physicians worldwide ${ }^{1}$.

Histopathological and bacteriological examination of the specimen obtained by pleural biopsy and a positive pleural fluid culture for Mycobacterium tuberculosis (MTB) are considered the gold standard for diagnosis².

Due to the invasiveness of pleural biopsy, more studies are trying to demonstrate the diagnostic accuracy of various scoring systems based on the association of clinical and laboratory tests ${ }^{3}$.

Nowadays, new diagnostic tests offer a quick diagnosis of tuberculous pleurisy, but the high cost limits their use in the current practice. Thus, these rapid diagnostic tests for tuberculous pleurisy are available only for research purposes.

High adenosine deaminase (ADA) levels detected in the pleural fluid are useful for the diagnosis of tuberculous pleurisy ${ }^{4}$. The diagnostic role of ADA in tuberculous pleurisy represents the main objective of many research studies. Since 1978, when ADA role in the diagnosis of tuberculous pleurisy was first described, routine assessment of ADA levels demonstrated high specificity and sensitivity ${ }^{5}$. granulome épithélioïde avec nécrose caséeuse en biopsie pleurale), entre 2011-2018, à l'Institut National de Pneumologie "Marius Nasta» Bucarest, Roumanie,

Résultats. Les valeurs de l'ADA étaient positivement et statistiquement significatives en corrélation avec les taux de lactate déshydrogénase (LDH) et de protéines dans le liquide pleural. En outre, la valeur moyenne de l'ADA a été augmentée chez les patients fébriles par rapport à ceux afébriles, statistiquement significative $(\mathrm{p}<0,05)$.

Conclusions. Les scores de probabilité (développés sur la base des caractéristiques biochimiques et cytologiques du liquide pleural, des paramètres cliniques et démographiques) ainsi que le contexte épidémiologique local peuvent contribuer à un diagnostic plus rapide, fiable, accessible et non invasif de la pleurésie tuberculeuse.

Mots-clés: ADA, LDH, pleurésie tuberculeuse, systèmes de notation.

ADA is a purine enzyme, a product of activated lymphocytes. It is the main catalytic in the enzymatic conversion of adenosine and deoxyadenosine to inosine and deoxyinosine ${ }^{6}$. ADA also plays an important role in the normal immune function ${ }^{7}$. ADA has two main iso-enzymes: ADA-1 and ADA-2. ADA-1 iso-enzyme is ubiquitous in humans, predominantly in T-lymphocytes, while ADA-2 coexists with ADA-1 only in monocytes-macrophages ${ }^{8}$. From the two isoforms, high ADA-2 pleural fluid levels demonstrated to be more accurate in the tuberculous pleurisy diagnosis. Even if the evaluation of ADA-2 level in the pleural fluid increases the diagnostic specificity, the costs of testing restrict this assessment only for research purposes. The ADA level in pleural fluid is easily tested through a rapid colorimetric assay ${ }^{5,9}$.

Different ADA cut-off values have been proposed for the diagnosis of tuberculous pleurisy, but its utility varies with the regional TB prevalence. In regions with an increased prevalence of tuberculosis, the association between high clinical suspicion, lymphocytic pleural effusion, ADA level higher than 40 IU/L carries a positive predictive value (PPV) of $98 \%$, so anti-tuberculous treatment can be initiated ${ }^{6,10}$.

In areas with a low prevalence of tuberculous disease, ADA level demonstrated to be useful to exclude the tuberculous etiology of the pleural effusions. In a recent case series, a cut-off value of ADA of $30 \mathrm{IU} / \mathrm{L}$ was shown to have a negative predictive value of $98.9 \%{ }^{11}$. 
A false low ADA level may be detected in the elderly, current smokers and HIV-positive patients. Despite some opinions about false negative ADA values in HIV-positive patients, ADA remains a biomarker with significant sensitivity in the diagnosis of tuberculous pleurisy in these patients ${ }^{12}$.

ADA values may be increased in other diseases, that are accompanied by the presence of pleural effusion, such as bacterial pleural infection, rheumatoid pleural effusions, mesothelioma, lung cancer and lymphoma. For these conditions, a complete assessment of the pleural fluid characteristics in the clinical context of the patient is required in order to have a more accurate diagnosis ${ }^{12-14}$.

The objective OF THE STUdY was to detect useful associations between ADA value in the pleural fluid and other data followed in our study (clinical, paraclinical, demographic data) in patients with confirmed tuberculous pleurisy.

\section{Material AND MEthods}

We conducted a retrospective study on $77 \mathrm{pa}$ tients diagnosed with tuberculous pleurisy. We collected data from the medical files of the patients found in the hospital archive. The database included patients diagnosed with tuberculous pleurisy hospitalized in The National Institute of Pneumology "Marius Nasta", Bucharest, Romania, between 2011 and 2018, a representative sample for a population of patients diagnosed with tuberculosis pleurisy in a specialized center. All the patients were confirmed with positive Mycobacterium tuberculosis (MTB) cultures in the pleural fluid or biopsy specimens and/ or specific histological aspect: epithelioid granuloma with caseous necrosis in pleural biopsy.

The access to the medical files of the patients included in the study was done with a previous permission granted by the hospital management and by the ethics committee (no. 24284/16.11.2018). On admission, each patient consented that his medical information may be used for medical research purposes.

The database was created using Excel database version 2007, part of Microsoft Office 365, (c) Microsoft Corporations. The analyzed parameters were divided into three categories (Table 1).

The following methodology was used: if the parameter of interest was a categorical variable, a Welch $\mathrm{T}$ bidirectional parametric test for two independent samples was used, as the criterion number has been met (eg $>30$ in each category). If the parameter of interest was a continuous variable, a Pearson $r$ correlation test was used for two continuous variables. Also, a descriptive statistical analysis was made for
Table 1. Demographic, clinical and biochemical data collected from patients included in the study.

\begin{tabular}{ccc}
\hline Demographic & $\begin{array}{c}\text { Clinical } \\
\text { findings }\end{array}$ & $\begin{array}{c}\text { Biochemical tests from the } \\
\text { pleural fluid }\end{array}$ \\
\hline gender & chest pain & ESR \\
\hline age & fever & leucocytes \\
\hline & sweats & lymphocytes (PF) \\
\hline & appetite loss & ADA (PF) \\
\hline weight loss & proteins (PF) \\
\hline & glucose (PF) \\
\hline & LDH (PF)
\end{tabular}

Legend. ESR-erythrocyte sedimentation rate; ADA-adenosine deaminase; LDH-lactate dehydrogenase; PF-pleural fluid.

Table 2. The general analysis of the clinical and biochemical data in patients from the group of study.

\begin{tabular}{lc}
\hline \multicolumn{1}{c}{ Variable } & Patients $($ No $=77)$ \\
\hline Age- mean \pm SD & $41.17 \pm 19.26$ \\
\hline Fever Yes - No (\%) & $40 / 77(51.94)$ \\
No- No $(\%)$ & $37 / 77(48.06)$ \\
\hline Leukocytosis Yes - No (\%) & $9 / 77(11.68)$ \\
No - No (\%) & $68 / 77(88.32)$ \\
\hline ESR- mean $\pm S D$ & $46.72 \pm 31.13$ \\
\hline Lymphocytes PF - mean \pm SD & $91.06 \pm 9.11$ \\
\hline Proteins PF - mean \pm SD & $5.11 \pm 0.89$ \\
\hline Glucose PF - mean \pm SD & $76.26 \pm 27.60$ \\
\hline LDH PF - mean \pm SD & $660.09 \pm 456.74$ \\
\hline ADA PF - mean \pm SD & $67.10 \pm 23.85$ \\
\hline
\end{tabular}

Legend. ESR-erythrocyte sedimentation rate; ADA-adenosine deaminase; LDH-lactate dehydrogenase; PF-pleural fluid; SD standard deviation

the variables followed in the study. For categorical variables the absolute and relative frequencies were calculated, while for the continuous variables the mean and standard deviation were calculated. All tests used were considered significant at a level $\alpha=$ 0.05 , which corresponds to a p-value less than 0.05 .

The data are presented as mean \pm standard deviation (SD) for continuous variables, and as no. (\%) for categorical variables.

For statistical analysis, we used the $\mathrm{R}$ program, version 4.0.2 (2020-06-22), Copyright (C) 2020, The R Foundation for Statistical Computing, R Core Team (2020). A: A language and environment for statistical computing. R Foundation for Statistical Computing, Vienna, Austria. URL https://www.R-project.org.

\section{Results}

We performed a statistical analysis of all the data presented in Table 1. The average age of the patients was 41.17 years. $51.94 \%$ of the patients presented 
Table 3. r Pearson pleural fluid

ADA correlations vs other continuous variables.

\begin{tabular}{|c|c|}
\hline Variable & $\begin{array}{c}\text { ADA PF } \\
\text { r Pearson [CI95\%] } \\
\text { p-value }\end{array}$ \\
\hline Age & $\begin{array}{c}-0.10[-0.32 \text { la } 0.12] \\
0.3593 \\
\end{array}$ \\
\hline Lymphocytes PF & $\begin{array}{c}-0.01[-0.25 \text { la } 0.21] \\
0.8860 \\
\end{array}$ \\
\hline Proteins PF & $\begin{array}{c}0.38 \text { [0.16 la } 0.56] \\
0.0007\end{array}$ \\
\hline ESR & $\begin{array}{c}0.14[-0.08 \text { la } 0.35] \\
0.2086 \\
\end{array}$ \\
\hline Glucose PF & $\begin{array}{c}-0.17\left[\begin{array}{c}-0.39 \text { la } 0.06] \\
0.1569\end{array}\right. \\
\end{array}$ \\
\hline LDH PF & 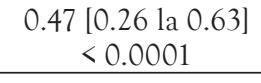 \\
\hline
\end{tabular}

Legend: PF-pleural fluid.

fever. On admission, only 9 patients $(11.68 \%)$ had leukocytosis. We calculated the mean values for the proteins, lymphocytes, glucose, $\mathrm{LDH}$, proteins and ADA levels from the pleural fluid (Table 2). The mean ADA value was $67.10 \mathrm{IU} / \mathrm{L}$.

As a result of statistical data processing, two positive correlations with statistical significance were
Table 4. The Welch $\mathrm{T}$ test comparison between ADA levels in the pleural fluid in both sexes.

\begin{tabular}{cccc}
\hline $\begin{array}{c}\text { Mean ADA } \\
\text { F }\end{array}$ & $\begin{array}{c}\text { Mean ADA } \\
\text { M }\end{array}$ & P-value & D [CI95\%] \\
\hline 65.30 & 69.14 & 0.4803 & $\begin{array}{c}-3.84[-14.64 \\
\text { la } 6.95]\end{array}$ \\
\hline
\end{tabular}

F-female; M-male; D-difference

Table 5. Welch T test comparison between ADA pleural levels in febrile vs. afebrile patients.

\begin{tabular}{cccc}
\hline $\begin{array}{c}\text { Mean } \\
\text { brile }- \text { fe- }\end{array}$ & $\begin{array}{c}\text { Mean } \\
\text { AD -afe- } \\
\text { brile }\end{array}$ & p-value & $\begin{array}{c}\text { Difference } \\
\text { [CI95\%] }\end{array}$ \\
\hline 73.08 & 60.63 & 0.0218 & $\begin{array}{c}12.45 \\
{[1.86-23.02]}\end{array}$ \\
\hline
\end{tabular}

obtained. One of them, a poor to average correlation, is represented by the correlation between the ADA and total protein values in the pleural fluid (Table 3 , Figure 1) $-r$ Pearson $=0.38$ with statistical significance $(\mathrm{p}<0.01)$. The other one, an average correlation, is represented by the correlation between the ADA value in the pleural fluid and the $\mathrm{LDH}$ value in the

\section{ADA PF vs proteins PF}

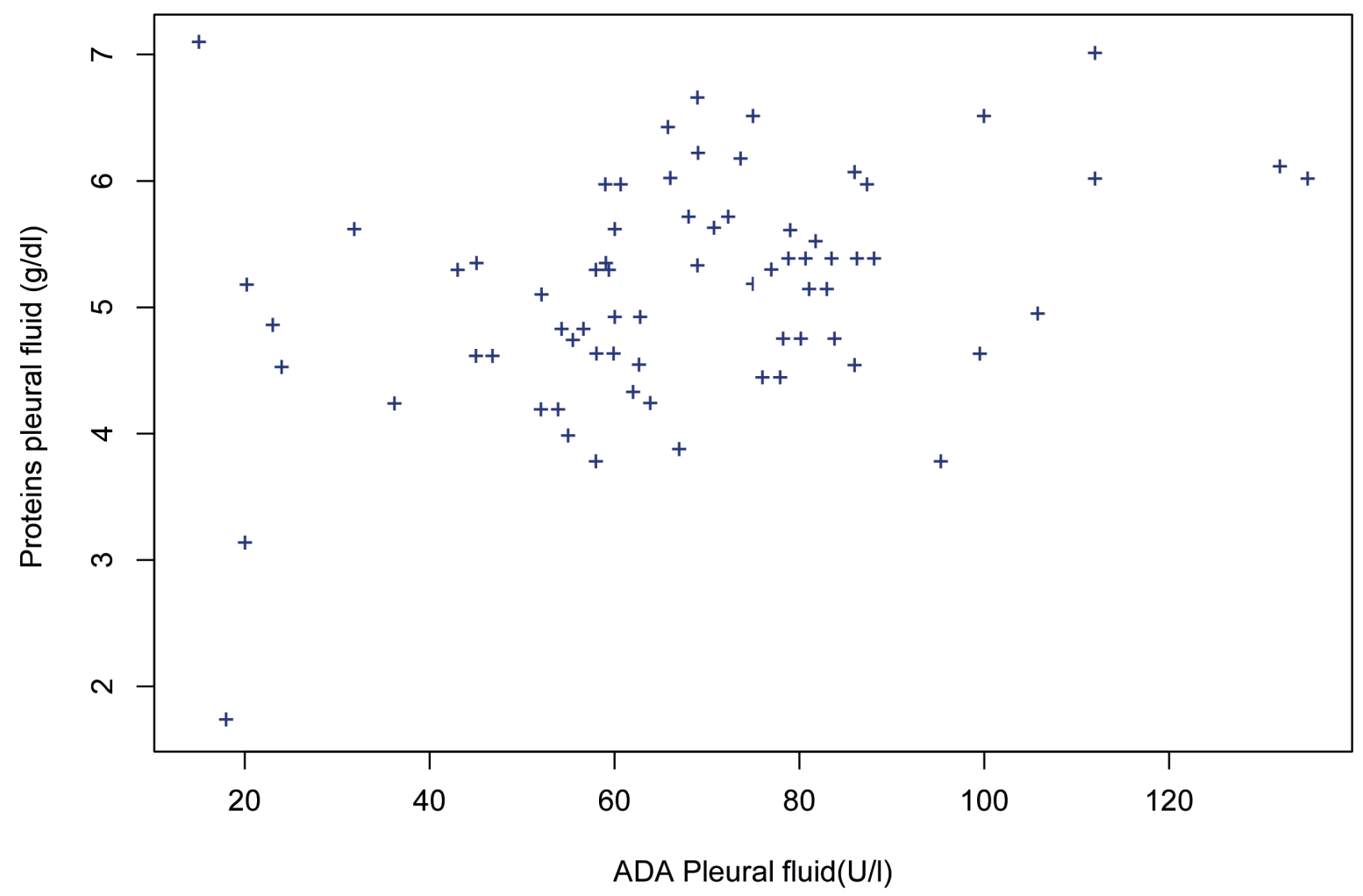

Figure 1. Positive correlation between pleural fluid ADA levels and pleural fluid proteins. 


\section{ADA PF vs LDH PF}

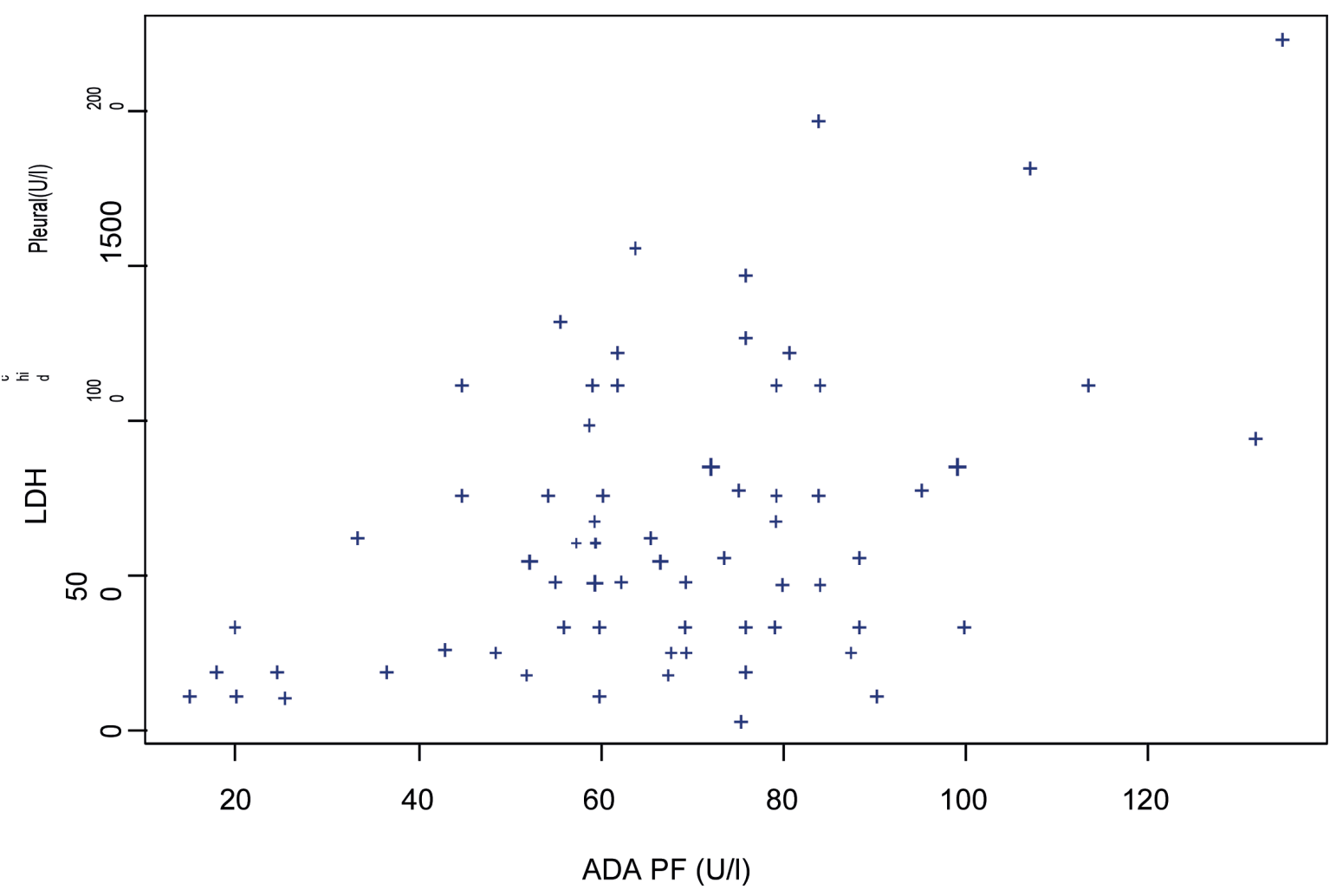

Figure 2. Positive correlation between ADA levels and LDH in the pleural fluid.

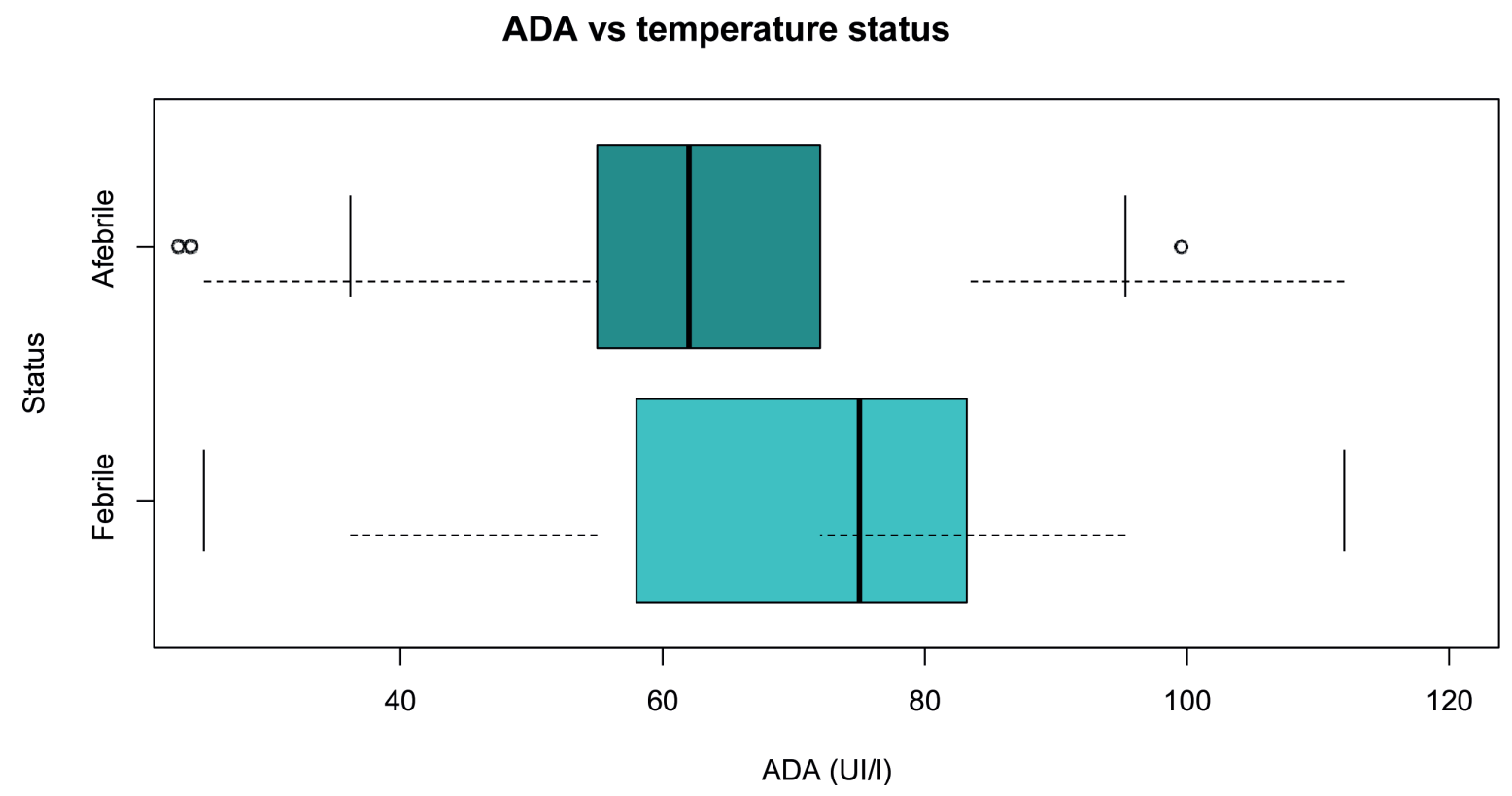

Figure 3. Positive statistical correlation between ADA level in the pleural fluid and temperature status.

pleural fluid (Table 3, Figure 2) $-\mathrm{r}$ Pearson $=0.47$ with statistical significance $(\mathrm{p}<0.01)$.

Following the comparative statistical analysis of the average value of ADA relative to the two sexes, no differences with statistical significance were observed ( $p>0.05)$.

Women had a mean ADA level in the pleural fluid of $65.30 \mathrm{IU} / \mathrm{L}$, while men had a mean ADA value 
in the pleural fluid of $69.14 \mathrm{IU} / \mathrm{L}$. The statistical analysis was performed using the Welch T test (Table 4).

Also, the Welch $T$ test was used to compare the ADA values in febrile patients versus afebrile patients. The mean ADA value was increased in febrile patients, the difference being statistically significant $(\mathrm{p}<0.05)$ (Table 5, Figure 3).

\section{Discussion}

The infection with MTB is considered one of the most common etiology of pleural disease, in some regions being considered the most common form of extrapulmonary tuberculosis ${ }^{13}$.

Tuberculous pleurisy is a disease with a global spread, its incidence being different from one region to another. In 2017, the World Health Organization reported 6.3 million newly discovered cases of tuberculosis, of which $16 \%$ were represented by extrapulmonary localizations ${ }^{15}$.

Romania is among the countries with a high incidence of tuberculosis cases, although during recent years there has been a slight decrease in the number of cases ${ }^{16}$.

In most cases, tuberculous pleurisy affects young adults, these patients being younger than those with pulmonary tuberculosis. No data are specified regarding the predominance of one of the sexes.

Currently, there are many methods used to facilitate the diagnosis of tuberculous pleurisy, but the gold standard for the diagnosis remains pleural biopsy with histological specific aspect (epithelioid granuloma with caseous necrosis) and positive MTB cultures from pleural fluid ${ }^{17}$.

Due to the invasiveness of the pleural biopsy, but also due to the prolonged time of MTB positivity of the cultures specimens (pleural fluid, pleural biopsy), a variety of biomarkers are intensively studied in order to identify the one with the highest diagnostic accuracy. Many authors are interested in evaluate the accuracy, the sensitivity and the specificity of some targeted biomarkers such as interferon- $\gamma, \mathrm{C}$ reactive protein, LDH, ADA, IL-6, and carcinoembryonic antigen ${ }^{18}$.

Many studies conducted so far have shown the diagnostic value of ADA in tuberculosis pleurisy. A systematic review and meta-analysis that included 12 studies demonstrated that the level of ADA in the pleural fluid plays an important role in the diagnosis of tuberculous pleurisy. There were analyzed 865 cases of tuberculous pleurisy from 12 studies and 1379 patients with non-tuberculous pleural effusions from 110 studies. The authors concluded that ADA represents an useful and promising marker for the diagnosis of tuberculous pleurisy, especially when ADA value is higher than $50 \mathrm{IU} / \mathrm{L}^{18}$.
Depending on the prevalence of tuberculosis by region, this biochemical marker shows a high negative predictive value. Its high specificity and sensitivity were also demonstrated by a prospective study that included 178 patients with different etiologies of pleural effusions (29 patients with tuberculous pleurisy, 63 with malignant pleural effusions, 40 with parapneumonic effusions, 18 patients with transudate, 5 patients with empyema, 19 patients with other causes of exudate and 4 patients with unknown cause of pleural effusion). The authors reported a cut-off value for ADA in the pleural fluid of $33.5 \mathrm{U} / \mathrm{L}$, with $93.1 \%$ sensitivity, $94.6 \%$ specificity, $77.1 \%$ positive predictive value, and $98.6 \%$ negative predictive value. The levels of ADA in the pleural fluid $<30.5 \mathrm{U} / \mathrm{L}$ suggested that the tuberculous etiology of pleural effusion is highly unlikely ${ }^{19,} 20$.

The value of the LDH/ADA ratio has been recently evaluated. A retrospective study, that included 72 cases of histologically confirmed tuberculous pleurisy and 47 patients with parapneumonic effusions, was developed in order to analyze the LDH and ADA levels in the pleural fluid and LDH/ADA ratios between the two groups. The study concluded that pleural fluid LDH/ADA ratio, which can be easily determined from routine biochemical analysis, is highly predictive of tuberculous pleurisy at a cut-off level of 16.20. This ratio may be useful in distinguishing between tuberculous pleurisy and parapneumonic effusions, with a reported sensitivity of $93.6 \%$ and a specificity of $93.1 \%$ in a high-incidence area of tuberculous effusions ${ }^{21}$.

In a similar research developed in a low-incidence area of tuberculous pleurisy (New Zealand), the authors identified an LDH/ADA threshold of < 15 as being highly predictive for tuberculous etiology of the effusions. The sensitivity was $89.1 \%$ and the specificity was $84.8 \%$. The association between an ADA level more than $30 \mathrm{IU} / \mathrm{L}$ and an LDH/ADA ratio less than 15 increased the specificity to $97.8 \%$, but reduced the sensitivity to $85.5 \%{ }^{22}$.

Conventional and individual tests used in the diagnosis of tuberculous pleurisy have their limitations. Due to these limitations, the researchers tried to develop composite diagnostic scores based on the results of several tests.

Given the fact that tuberculous pleurisy and malignant pleural effusions have similar clinical manifestations ${ }^{23}$, a model of scoring system was proposed by a group of researchers. They developed a retrospective study for 9 years, including 106 patients with tuberculous pleurisy and 286 patients with malignant pleural effusions. The authors created two models of scoring systems. Both models were developed based on the same markers, with one exception, model 2 
was created without ADA pleural fluid levels. They obtained the model 1 using four predictive markers: level of ADA in the pleural fluid $\geq 40 \mathrm{U} / \mathrm{L}$, age, temperature and pleural fluid red blood cell count. The model 2 was created by adding to model 1 other 3 variables: no history of malignancy, pleural fluid proteins, and pleural fluid to serum LDH ratio, but without ADA levels. The summated scores of $>$ or 5 in model 1 and > or 6 in model 2 demonstrated to have a high sensitivity (95\% and $97 \%$ ), and high specificity (94\% and $91 \%$ ) in distinguishing tuberculous from malignant effusions, respectively ${ }^{24}$.

Another group of researchers also suggested a model of scoring system based on clinical findings and biological markers. In their study, the analyzed parameters were ADA activity, IFN- $\gamma$ levels and polymerase chain reaction (PCR). The biomarkers were tested on the same sample for the diagnosis of microbiologically and/or histologically confirmed tuberculous pleurisy. The sensitivity and specificity of ADA activity were $88 \%$, respectively $86 \%$, the sensitivity and the specificity for IFN- $\gamma$ levels were $86 \%$, respectively $97 \%$. For the PCR values, a sensitivity of $74 \%$ and a specificity of $90 \%$ were reported. The authors concluded that the combinations of ADA activity, PCR values and IFN- $\gamma$ levels from pleural fluid increase the sensitivity and specificity compared with individual use, so it is provided the basis for a rapid and efficient diagnosis of pleural tuberculosis in different clinical settings ${ }^{25}$.

A latest model of composite scoring system was proposed by a group of researchers from Buenos Aires, Argentina. They developed a model measuring glucose, $\mathrm{LDH}$, proteins, lymphocytes, and $\mathrm{pH}$ in the pleural fluid. The ADA level in the pleural fluid was not measured because of the low availability of the test in their setting. Their aim was to develop a score to differentiate tuberculous pleurisy from malignant pleural effusions. The authors prospectively analyzed 82 patients, 47 patients with confirmed tuberculous pleurisy and 35 patients with malignant pleural effusion. A cut-off of eight points was required to achieve 93.5\% sensitivity, $78 \%$ specificity, and a likelihood ratio of 4.26 to distinguish tuberculous pleurisy from malignant pleural effusion ${ }^{26}$.

The limits of the study. Our study presents some limitations that must be acknowledged. Due to the retrospective type of the study, one of the limitations come from the incomplete data filled in the patient's file. We analyzed data from a single-center population. To realize a diagnostic scoring system, it is necessary to analyze a comparative group of patients with pleural effusions with other etiologies than the tuberculous etiology.

\section{Conclusions}

In our patients diagnosed with tuberculous pleurisy, the ADA values were positively and statistically significant correlated with LDH and protein levels in the pleural fluid. The mean ADA value is statistically significant $(\mathrm{p}<0.05)$ increased in febrile patients as compared with afebrile ones. Further studies on correlations between pleural fluid biochemical characteristics and various clinical and epidemiological data are necessary to obtain new correlations useful for the positive diagnosis of tuberculous pleurisy. Probability scores (developed on the biochemical and cytological characteristics of the pleural fluid, clinical and demographic parameters) together with the local epidemiological context may contribute to a faster, reliable, accessible and non-invasive diagnosis of tuberculous pleurisy.

\section{Author contribution:}

A.P.D, D.C.Z, S.D-R, C.L.T, A.M.B equally contributed to the design of the study, to patient recruitment, analysis of the findings and writing of the manuscript. All authors were responsible for the collection and assembly of the articles/published data, and their inclusion and interpretation in this article. All authors contributed to the critical revision of the manuscript for valuable intellectual content. All authors have read and agreed to the published version of the manuscript.

\section{Compliance with Ethics Requirements:}

„The authors declare no conflict of interest regarding this article"

"The authors declare that all the procedures and experiments of this study respect the ethical standards in the Helsinki Declaration of 1975, as revised in 2008(5), as well as the national law. Informed consent was obtained from all the patients included in the study"

"No funding for this study“

\section{References}

1. Jeon D. Tuberculous pleurisy: an update. Tuberc Respir Dis (Seoul). 2014;76(4):153-159.

2. Skouras VS, Kalomenidis I. Pleural fluid tests to diagnose tuberculous pleuritis. Curr Opin Pulm Med. 2016;22(4):367377.

3. Trajman A, Pai M, Dheda K, et al. Novel tests for diagnosing tuberculous pleural effusion: what works and what does not? Eur Respir J. 2008;31(5):1098-1106.

4. Jiang J, Shi HZ, Liang QL, Qin SM, Qin XJ. Diagnostic value of interferon- $\gamma$ in tuberculous pleurisy: A meta-analysis. Chest. 2007;131(4):1133-1141.

5. Valdés L, San José E, Alvarez D, Valle JM. Adenosine deaminase (ADA) isoenzyme analysis in pleural effusions: 
diagnostic role, and relevance to the origin of increased ADA in tuberculous pleurisy. Eur Respir J. 1996;9(4):747-751.

6. Shaw JA, Irusen EM, Koegelenberg CFN. Pleural effusion: tuberculous effusion. In: Reference Module in Biomedical Sciences. Elsevier; 2019.

7. Broaddus VC, Light RW. Pleural effusion. In: Murray and Nadel's Textbook of Respiratory Medicine. Elsevier; 2016:13961424.e10.

8. Gakis C. Adenosine deaminase (ADA) isoenzymes ADA1 and ADA2: diagnostic and biological role. Eur Respir J. 1996;9(4):632-633.

9. Shaw JA, Irusen EM, Diacon AH, Koegelenberg CF. Pleural tuberculosis: a concise clinical review. Clin Respir J. 2018;12(5):1779-1786.

10. Liang QL, Shi HZ, Wang K, Qin SM, Qin XJ. Diagnostic accuracy of adenosine deaminase in tuberculous pleurisy: A meta-analysis. Respir Med. 2008;102(5):744-754.

11. Sivakumar P, Marples L, Breen R, Ahmed L. The diagnostic utility of pleural fluid adenosine deaminase for tuberculosis in a low prevalence area. Int J Tuberc Lung Dis. 2017;21(6):697-701.

12. Lee SJ, Kim HS, Lee SH, et al. Factors influencing pleural adenosine deaminase level in patients with tuberculous pleurisy. Am J Med Sci. 2014;348(5):362-365.

13. Light RW. Tuberculous pleural effusion. Turk Toraks Derg. 2015;16(1):1-9.

14. Light RW. Update on tuberculous pleural effusion. Respirology. 2010;15(3):451-458.

15. Antonangelo L, Faria CS, Sales RK. Tuberculous pleural effusion: diagnosis \& management. Expert Rev Respir Med. 2019;13(8):747-759.

16. Golli AL, Nitxu MF, Turcu F, Popescu M, Ciobanu-Mitrache L, Olteanu M. Tuberculosis remains a public health problem in Romania. Int J Tuberc Lung Dis. 2019;23(2):226-231.
17. Bibby AC, Maskell NA. Pleural biopsies in undiagnosed pleural effusions; Abrams vs image-guided vs thoracoscopic biopsies. Curr Opin Pulm Med. 2016;22(4):392-398.

18. Gui X, Xiao H. Diagnosis of tuberculosis pleurisy with adenosine deaminase (ADA): A systematic review and meta-analysis. Int J Clin Exp Med. 2014;7(10):3126-3135.

19. Saiphoklang N, Kanitsap A, Nambunchu A. Differences in clinical manifestations and pleural fluid charateristics between tuberculous and malignant pleural effusions. Southeast Asian J Trop Med Public Health. 2015;46(3):496-503.

20. Reechaipichitkul W, Kawamatawong T, Teerajetgul Y, Patjanasoontorn B. Diagnostic role of pleural fluid adenosine deaminase in tuberculous pleural effusion. Southeast Asian J Trop Med Public Health. 2001;32(2):383-389.

21. Wang J, Liu J, Xie X, Shen P, He J, Zeng Y. The pleural fluid lactate dehydrogenase/adenosine deaminase ratio differentiates between tuberculous and parapneumonic pleural effusions. BMC Pulm Med. 2017;17(1):168.

22. Blakiston M, Chiu W, Wong C, Morpeth S, Taylor S. Diagnostic performance of pleural fluid adenosine deaminase for tuberculous pleural effusion in a low-incidence setting. J Clin Microbiol. 2018;56(8):e00258-18.

23. Paraschiv B, Dediu G, Iancu A, Bratu O, Diaconu C. Superior vena cava syndrome - review. Arch Balk Med Union. 2017;52(1):39-43.

24. Porcel JM, Vives M. Differentiating tuberculous from malignant pleural effusions: a scoring model. Med Sci Monit. 2003;9(5):CR175-80.

25. Villegas M V., Labrada LA, Saravia NG. Evaluation of polymerase chain reaction, adenosine deaminase, and interferon- $\gamma$ in pleural fluid for the differential diagnosis of pleural tuberculosis. Chest. 2000;118(5):1355-1364.

26. González A, Fielli M, Ceccato A, Luna C. Score for differentiating pleural tuberculosis from malignant effusion. Med Sci. 2019;7(3). 